\title{
UTILIZAÇÃO DO FRAMEWORK UEF-WEB NO DESENVOLVIMENTO DE UMA APLICAÇÃO WEB ERGONÔMICA
}

\author{
Diego Santana Silveira, PROCC/UFS, IFS, di.diegosilveira@gmail.com \\ Henrique Nou Schneider, PROCC/UFS, IFS, hns@terra.com.br
}

Resumo. Construir interfaces com maior qualidade de uso é um desafio que tem se tornado crítico para o sucesso das aplicações Web junto às empresas e aos usuários. Pesquisas foram realizadas, mas há lacunas quanto ao fornecimento de soluções integradas de suporte à usabilidade para as diversas fases de um projeto de aplicação Web. Diante disso, este artigo apresenta o Framework UEF-WEB como instrumento de apoio à usabilidade. Este framework, composto por fases, atividades, recursos e artefatos, tem por objetivo ajudar as organizações a introduzir recursos de usabilidade no processo de planejamento, desenvolvimento e avaliação de interfaces, com vistas à ampliação da qualidade de uso destas. Os resultados do estudo de caso forneceram indícios da viabilidade de utilização deste framework no suporte à usabilidade.

Palavras-chave: usabilidade, processo de desenvolvimento de interfaces, framework, aplicações web.

\section{USING UEF-WEB FRAMEWORK IN THE DEVELOPMENT OF A WEB APPLICATION ERGONOMIC}

\begin{abstract}
Build interfaces with higher quality of use is a challenge that has become critical to the success of Web applications with companies and users. Researches have been conducted, but there are gaps in the provision of integrated support in usability in various stages of a Web application project. Thus, this paper proposes the UEF-WEB Framework as instrument to support the usability. This framework, composed of stages, activities, resources and artifacts, has the objective to help organizations to introduce usability features in the planning, development and evaluation of Web application interfaces process, in order to expand the use of these quality. The case study results provided evidence of the feasibility of using this framework in support usability.
\end{abstract}

Keywords: usability, development process interfaces, framework, web applications.

\section{Introdução}

$\mathrm{Na}$ última década, a demanda por aplicações $W e b$ cresceu ligeiramente para atender às variadas necessidades das pessoas e organizações, a exemplo do apoio à execução de atividades laborais, mercadológicas, educacionais e de comunicação, dentre outras. Essa expansão pode ser notada não somente em relação à quantidade e diversidade, como também no nível de complexidade das aplicações.

A simplicidade de acesso a informações e serviços fomentada pela Web tem possibilitado a utilização em larga escala dessas aplicações na sociedade, como suporte à realização de atividades cotidianas. Mais do que apoiar essas atividades, as aplicações $W e b$ estão influenciando a forma como pessoas e organizações procuram informações, efetuam compras, comunicam-se, emitem opiniões, estudam e até mesmo realizam atividades de entretenimento (PRESSMAN; LOWE, 2009).

Os benefícios decorrentes do uso de aplicações $W e b$ são inúmeros e vão desde a automatização, maior agilidade na execução de tarefas, facilidades e melhorias no 
processo de comunicação até a diminuição de custos (PRESSMAN, 2011). Entretanto, problemas relativos à ergonomia das interfaces dessas aplicações têm sido observados frequentemente, a exemplo de alta complexidade, baixa flexibilidade, facilidade e eficácia de uso (NIELSEN; LORANGER, 2007).

A Educação à Distância (e-learning), por exemplo, para realizar seu objetivo maior, isto é, que o aluno aprenda, depende da qualidade ergonômica dos Ambientes Virtuais de Aprendizagem (AVA) utilizados como plataforma para a ministração dos cursos, tanto no que diz respeito à dimensão da funcionalidade do software quanto na qualidade das interfaces utilizadas pelo aprendiz para ter acesso ao conhecimento, bem como interagir com o professor e demais colegas através do AVA.

A ergonomia é definida como a ciência que estuda a adequação do trabalho às características do ser humano para garantir que sistemas e dispositivos estejam adaptados ao modo como o usuário pensa, comporta-se e exerce atividades laborais (CYBIS; BETIOL; FAUST, 2010). No tocante à Interação Humano-Computador (IHC), a ergonomia pode ser mensurada pelo nível de usabilidade e acessibilidade fornecido. A usabilidade é entendida como um atributo de qualidade associado à facilidade e eficiência de uso e ao grau de satisfação em realizar algo (ISO, 2010). A acessibilidade, por seu turno, está relacionada à capacidade dos sistemas computacionais serem utilizados por indivíduos com alguma limitação física.

$\mathrm{Na}$ atualidade, com o crescente nível de informatização das atividades do ser humano, os requisitos de usabilidade e de acessibilidade tornaram-se indispensáveis, visto que, imprimem valor a serviços e produtos cada vez mais utilizados na sociedade contemporânea como o e-learning e e-commerce. Assim, se faz importante desenvolver aplicações à luz das necessidades dos usuários e em conformidade a estes requisitos.

Diante disso, métodos, técnicas e ferramentas foram propostos para auxiliar no desenvolvimento de interfaces ergonômicas (HART; PORTWOOD, 2009; AHMAD; SULAIMAN; JOHARI, 2010; FERRE; BEVAN, 2011; FERNANDES; CONTE; BONIFÁCIO, 2012). Todavia, percebe-se que há lacunas quanto ao fornecimento de soluções integradas de suporte à usabilidade. Existem também organizações que não utilizam recursos de usabilidade (INSFRAN; FERNANDEZ, 2008) por desconhecer, não dispor de orçamento e enfrentar dificuldades na seleção e adoção desses recursos (CONTE, 2009; CYBIS; BETIOL; FAUST, 2010).

$\mathrm{Na}$ esteira dessa discussão, o artigo apresenta um framework como instrumento de apoio à usabilidade com o intuito de auxiliar as organizações a introduzir, de maneira sistemática e facilitada, recursos de usabilidade no processo de desenvolvimento de interfaces, com vistas à ampliação da qualidade de uso das interfaces. Para isso, foi realizada uma pesquisa aplicada com abordagem qualitativa e natureza exploratória, tendo por base os procedimentos técnicos de revisão bibliográfica e estudo de caso.

Este artigo está organizado da seguinte forma: a primeira seção apresenta o tema do trabalho; a segunda seção discute a usabilidade na Interação HumanoComputador (IHC); a terceira seção apresenta o framework desenvolvido para apoiar a construção de interfaces ergonômicas; a quarta seção discute os resultados do estudo de caso conduzido para aplicar este framework; a quinta seção apresenta as considerações finais e oportunidades de trabalhos futuros.

\section{Usabilidade na Interação Humano-Computador (IHC)}

A usabilidade é considerada um dos critérios mais relevantes para aceitação de aplicações de software interativas, especialmente de aplicações Web (HITZ et al., 2006). O termo usabilidade é definido pela International Standards Organization (ISO) 
como a medida na qual um sistema, produto ou serviço pode ser utilizado por usuários específicos para alcançar determinados objetivos com eficácia, eficiência e satisfação em um dado contexto de uso (ISO, 2010). A medida de eficácia diz respeito à exatidão com que objetivos específicos são alcançados pelos usuários. A medida de eficiência está associada aos recursos consumidos para atingir os objetivos definidos. A medida de satisfação mede a ausência de desconforto e a atitude positiva do usuário ao utilizar um produto. $\mathrm{O}$ contexto de uso envolve usuários, tarefas, equipamentos e ambiente.

Uma definição alternativa para o termo usabilidade é a inexistência de obstáculos (problemas) que impeçam os usuários de realizar e completar com sucesso suas tarefas em um sistema (SKOV; STAGE, 2005). Na prática, esses problemas se revelam na forma de interfaces complexas, com erros, com baixa flexibilidade, facilidade e eficácia de uso (MATERA et al., 2006; NIELSEN; LORANGER, 2007).

De fato, não é uma tarefa fácil desenvolver interfaces ergonômicas e adequadas ao ser humano, pois as interfaces apresentam-se como sistemas abertos onde os usuários são agentes ativos e com comportamento não definido (CYBIS; BETIOL; FAUST, 2010). Diante disso, surgiu a área de conhecimento IHC que visa compreender e planejar as formas de interação entre indivíduos e dispositivos computacionais, de modo que as necessidades dos indivíduos sejam atendidas com efetividade (GALITZ, 2003).

A usabilidade na IHC também pode ser entendida como o resultado de uma equação composta por elementos como a experiência do usuário com a tecnologia, a eficiência do hardware, a natureza da tarefa e o ambiente onde se desenvolve a tarefa (SCHNEIDER, 2008). A visão que o projetista de software possui sobre o problema a ser modelado muitas vezes não é a visão que o usuário tem para solucionar o problema, o que acaba dificultando a interação entre o usuário e o sistema. Esse descompasso de entendimento precisa ser equacionado para que as concepções dos projetistas e dos usuários acerca da solução de um problema estejam devidamente alinhadas.

Diferentemente da Engenharia de Software que direciona a atenção para a lógica de funcionamento do software, a Engenharia de Usabilidade está focada na interface do software com o usuário. Em suma, a Engenharia de Usabilidade é definida como o processo de construção de interfaces que visa promover facilidade de uso e de aprendizado e satisfação dos usuários ao interagir com um sistema (ROCHA; BARANAUSKAS, 2003). O objetivo da Engenharia de Usabilidade é o de auxiliar as organizações na construção de software de maneira que o modelo mental do desenvolvedor esteja de acordo com o modelo mental do usuário (SCHNEIDER, 2008). Em outras palavras, as interfaces devem refletir o modo de pensar e agir dos usuários.

Estas interfaces, agora desenvolvidas sob a perspectiva de uma lógica de operação, são componentes de um sistema interativo de software constituído por estruturas de diálogos e apresentações, que fornecem informações e controles para o usuário realizar tarefas. É possível até mesmo afirmar que um desenvolvedor tem mais chances de obter sucesso ao construir o núcleo funcional de um software do que interfaces com o usuário. A explicação para isso se encontra no fato de que o núcleo funcional é um sistema fechado, com comportamento previamente estabelecido (CYBIS; BETIOL; FAUST, 2010).

Diante dessas dificuldades, foram propostos nas últimas décadas métodos, técnicas e modelos de ciclo de vida para a Engenharia de Usabilidade. Os métodos e técnicas desenvolvidos podem ajudar as organizações a realizar diversas atividades, a exemplo de levantamento e registro de informações, especificação de requisitos, projeto e desenvolvimento de versões de interfaces, avaliação de soluções, entre outras. Os modelos de ciclo de vida, por sua vez, trazem orientações por meio de princípios e atividades de projeto que ajudam na obtenção de interfaces com maior qualidade de uso. 
Tais modelos procuram suportar o desenvolvimento de soluções de interfaces mais consistentes, que respondam positivamente às expectativas dos usuários na execução de suas tarefas. Todavia, esses modelos não fornecem maiores informações sobre instrumentos e artefatos de apoio às atividades neles previstas o que, na prática, dificulta a realização dessas atividades e, por conseguinte, o alcance dos objetivos definidos.

Além da adoção de princípios e diretrizes de projeto, o uso de frameworks no processo de desenvolvimento de software tem contribuído para ampliar a qualidade das aplicações construídas (SOMMERVILLE, 2007; PRESSMAN, 2011). Tomhave (2005) define framework como um construtor fundamental que estabelece pressupostos, conceitos, valores e práticas e que inclui diretrizes para a execução propriamente dita.

Frameworks oferecem uma estrutura comum para um domínio de aplicações ao promover o reuso de conteúdo conceitual do domínio de um software ou da solução de um problema (GIMENES; HUZITA, 2005). O reuso proporcionado com frameworks não se restringe apenas à implementação, reflete-se também na análise e no projeto de um software. A adoção de frameworks permite organizar e explicar, de forma gráfica e/ou textual, aspectos elementares a serem estudados como conceitos, constructos e suas relações. À luz do discutido, será apresentado, na próxima seção, um framework como instrumento de apoio ao desenvolvimento de aplicações ergonômicas.

\section{Framework UEF-WEB}

O Framework de apoio à Engenharia de Usabilidade para aplicações WEB (UEF-WEB) consiste em um arcabouço sucinto de fases, atividades, recursos e artefatos de suporte à usabilidade. A utilização deste framework em organizações busca promover a adoção sistematizada de recursos de usabilidade e, como isso, elevar a qualidade ergonômica das interfaces computacionais desenvolvidas.

A ideia central deste framework é a execução, de forma evolutiva e com a participação dos usuários, de um conjunto de fases, atividades, recursos e artefatos de apoio à usabilidade, de modo a garantir que, a cada ciclo de desenvolvimento, a aplicação Web corresponda melhor às expectativas e necessidades dos usuários. Tal estratégia, na qual o paradigma de desenvolvimento de interfaces com usuários parte de sucessivos ciclos de análise, concepção e testes, segue os preceitos da norma ISO 9241 210 (ISO, 2010). A construção deste framework foi realizada através de pesquisa bibliográfica e análise de propostas relacionadas à usabilidade.

O Framework UEF-WEB é composto por três fases: Planejamento, Desenvolvimento e Avaliação. Cada fase possui objetivos específicos dentro do processo de desenvolvimento de interfaces e demanda a realização de uma série de atividades que, por sua vez, são apoiadas por recursos e artefatos providos pela Engenharia de Usabilidade. É importante destacar que durante o processo de construção de um software podem ser realizados vários ciclos de atividades. Em cada ciclo, atividades previstas nas fases do framework são executadas com o propósito de atingir os objetivos definidos e os resultados produzidos em cada fase servem de entrada para a fase seguinte. Nas próximas subseções, são apresentadas as três fases deste framework, assim como as atividades, recursos e artefatos estabelecidos.

\subsection{Fase de Planejamento}

A Fase de Planejamento estabelece como objetivos principais a especificação do contexto de uso e dos requisitos de usabilidade das interfaces da aplicação Web. A atividade de Análise e Especificação do Contexto de Uso visa levantar informações 
acerca dos usuários, das tarefas e do ambiente onde será utilizada a aplicação $W e b$. As informações produzidas nesta atividade constituem o contexto de uso e correspondem a insumos importantes para as demais atividades deste framework.

Diversos recursos podem ser empregados no processo de coleta de informações sobre o contexto de uso e definição de requisitos de usabilidade de uma aplicação. Este framework estabelece a utilização dos recursos de Entrevista e Reunião. É importante destacar que estes recursos, apesar de serem técnicas elementares de análise, fornecem meios que permitem obter, de forma simples e rápida, as informações requeridas nesta fase, bem como dirimir eventuais dúvidas e/ou inconsistências.

Concluída a atividade de Análise e Especificação do Contexto de Uso, deve ser executada a atividade de Especificação de Requisitos de Usabilidade. Esta atividade objetiva definir com mais detalhes os requisitos de usabilidade da aplicação. Estes requisitos correspondem a níveis de qualidade a serem alcançados pelos usuários quando da interação com as interfaces. Para auxiliar esta atividade, foi definida a Tabela de Especificação de Requisitos de Usabilidade apresentada na Tabela 1.

Tabela 1 - Tabela de Especificação de Requisitos de Usabilidade.

\begin{tabular}{|c|c|c|c|c|c|}
\hline ID & $\begin{array}{c}\text { Atributo de } \\
\text { usabilidade }\end{array}$ & $\begin{array}{c}\text { Tipo de } \\
\text { usuário }\end{array}$ & $\begin{array}{c}\text { Instrumento } \\
\text { de medida }\end{array}$ & $\begin{array}{c}\text { Valor a ser } \\
\text { medido }\end{array}$ & $\begin{array}{c}\text { Nível de } \\
\text { desempenho } \\
\text { almejado }\end{array}$ \\
\hline & & & & & \\
\hline & & & & & \\
\hline
\end{tabular}

Em relação à Tabela 1, tem-se: ID diz respeito a um identificador utilizado para referenciar cada atributo de usabilidade; Atributo de usabilidade corresponde a características de usabilidade definidas como parâmetros de qualidade de uso; Tipo de usuário se refere aos perfis de usuários que vão realizar tarefas por meio das interfaces; Instrumento de medida relaciona-se ao mecanismo empregado para coletar os valores de um atributo de usabilidade específico; Valor a ser medido diz respeito ao tipo de dado ou medida que deve ser coletada sobre os atributos de usabilidade; Nível de desempenho almejado, por sua vez, refere-se a metas de desempenho tidas como alvo para os atributos de usabilidade.

As informações obtidas nas duas atividades da Fase de Planejamento devem ser registradas no artefato Contexto de Uso e Requisitos de Usabilidade. Este artefato é constituído de três seções principais, são elas: a primeira seção apresenta informações acerca do objetivo do artefato; a segunda seção reúne informações sobre visão, metodologia, programação de atividade, usuários, tarefas e ambiente de uso; a terceira seção apresenta informações sobre diretrizes e requisitos de usabilidade. Na próxima subseção, será apresentada a Fase de Desenvolvimento.

\subsection{Fase de Desenvolvimento}

A Fase de Desenvolvimento tem por objetivos projetar e construir as interfaces da aplicação $W e b$. As atividades de Projeto e de Implementação presentes nesta fase são atividades típicas da Engenharia de Software relacionadas a aspectos de modelagem e de construção. Durante a atividade de Projeto, este framework estabelece a utilização do recurso de Prototipação. O uso de protótipos permite não somente explorar soluções alternativas de interfaces junto aos usuários, mas também avaliar antecipadamente estas soluções visando reduzir os riscos do projeto e os encargos de mudanças futuras. Como forma de apoiar este recurso, foi definido o artefato Formulário de Feedback apresentado no Quadro 1. 
Quadro 1 - Artefato Formulário de Feedback.

\begin{tabular}{|l|c|c|c|}
\hline Tipo de usuário & $\begin{array}{c}\text { Identificação da } \\
\text { interface }\end{array}$ & $\begin{array}{c}\text { Descrição do } \\
\text { problema/necessidade/sugestão }\end{array}$ & $\begin{array}{c}\text { Nível de prioridade } \\
\text { (baixo, médio ou alto) }\end{array}$ \\
\hline & & & \\
\hline & & & \\
\hline
\end{tabular}

Sobre o Quadro 1, observa-se que: Tipo de usuário diz respeito aos perfis de usuários que realizam as tarefas nas interfaces; Identificação da interface corresponde a uma descrição da interface analisada; Descrição do problema/necessidade/sugestão se refere aos relatos fornecidos pelos usuários acerca de problemas, necessidades ou sugestões de melhorias percebidas acerca das interfaces; Nível de prioridade (baixo, médio ou alto) está relacionado ao grau de prioridade atribuído pelo usuário para correção de um problema, atendimento de uma necessidade ou sugestão de melhoria. Essas informações ajudam a equipe de desenvolvimento a sanar dúvidas, proceder na correção de problemas e suprir necessidades identificadas nas interfaces da aplicação.

Para auxiliar na descoberta de problemas nas interfaces desenvolvidas, este framework estabelece a utilização do recurso de Inspeção de Usabilidade Baseada em Perspectivas. Neste framework, a adoção deste recurso será através da técnica Web Design Perspectives-Based Usability Evaluation (WDP) proposta por Conte (2009). Esta técnica combina três perspectivas de projeto $W e b$ (apresentação, conceituação e navegação) com as dez heurísticas de usabilidade de Nielsen (1994). Para apoiar a aplicação desta técnica pela equipe de desenvolvimento, foi definido o artefato Formulário de Inspeção apresentado no Quadro 2.

Quadro 2 - Artefato Formulário de Inspeção.

\begin{tabular}{|c|c|c|c|c|}
\hline Avaliador & Tarefa & $\begin{array}{c}\text { Classificação do } \\
\text { problema } \\
\text { (Perspectiva.Heurística) }\end{array}$ & $\begin{array}{c}\text { Descrição do } \\
\text { problema } \\
\text { (cosmético, } \\
\text { leve, grave ou } \\
\text { catastrófico) }\end{array}$ \\
\hline & & & & \\
\hline
\end{tabular}

Com relação ao Quadro 2, observa-se que: Avaliador se refere ao integrante da equipe de desenvolvimento que está realizando a inspeção de usabilidade; Tarefa diz respeito a uma descrição sucinta da interface e da tarefa analisadas; Classificação do problema (Perspectiva.Heurística) corresponde à categorização do problema através da relação Perspectiva.Heurística; Descrição do problema se refere à apresentação do problema/violação identificado; Severidade (cosmético, leve, grave ou catastrófico) está associado ao nível de gravidade atribuído para o problema de usabilidade.

As inspeções de usabilidade realizadas durante a atividade de Implementação seguem um roteiro composto por três etapas: (i) sessões de inspeção individuais; (ii) consolidação das inspeções; (iii) seleção dos problemas a serem corrigidos. Cabe ainda destacar que a própria equipe de desenvolvimento executa as inspeções após treinamento sobre a técnica WDP. Este fato reduz a dependência de especialistas em usabilidade na avaliação de interfaces. A seguir, será apresentada a Fase de Avaliação.

\subsection{Fase de Avaliação}

A Fase de Avaliação tem por objetivo mensurar a satisfação dos usuários ao realizar tarefas e interagir com as interfaces da aplicação. Nesta fase, é realizada a 
atividade de Teste de Usabilidade cujo objetivo é colocar os usuários em contato direto com a aplicação desenvolvida para que possam interagir e relatar as suas percepções, bem como nível de satisfação em relação às interfaces utilizadas.

A coleta de informações acerca da satisfação dos usuários quanto às interfaces da aplicação é feita com o auxílio do recurso de Questionário. Neste framework, foi adotado o questionário System Usability Scale (SUS) proposto por Brooke (1986), acrescido de questões fechadas e abertas para obter informações acerca do perfil e da percepção dos usuários, respectivamente. Três questões fechadas foram adicionadas visando observar a evolução do grau de satisfação dos usuários sob os critérios de orientação sexual, idade e nível de habilidade na Internet. Duas questões abertas foram incluídas com o propósito de reunir informações sobre características positivas e negativas da aplicação em avaliação.

O questionário SUS é composto por dez questões e utiliza escala Likert de cinco pontos com valores entre 1 (discordo completamente), 2 (discordo), 3 (neutro), 4 (concordo) e 5 (concordo completamente). A pontuação neste questionário é calculada da seguinte forma (BROOKE, 1986): (i) cada questão possui valores de 0 a 4; (ii) a contribuição das questões ímpares é calculada pela posição na escala Likert menos 1; (iii) a contribuição das questões pares é de 5 menos a posição na escala Likert; (iv) a soma das contribuições das dez questões é multiplicada por 2,5 para se chegar à pontuação final, que varia de 0 a 100.

Ainda sobre esta pontuação, a média de 70 pontos tem se mantido em diferentes aplicações do SUS (BANGOR; KORTUM; MILLER, 2009). Diante disso, esta média de pontuação foi utilizada como medida de usabilidade para avaliar a satisfação dos usuários em relação à aplicação $W e b$. Na próxima seção, será apresentado o estudo de caso realizado para aplicar este framework no suporte à usabilidade.

\section{Estudo de Caso}

Este estudo de caso foi executado com o intuito de observar a viabilidade de utilização do Framework UEF-WEB em um projeto de desenvolvimento de interfaces de uma aplicação $W e b$, denominada AvWeb. Esta aplicação tem como função principal fornecer um questionário (checklist) de apoio à avaliação de usabilidade de interfaces computacionais. Para isso, foram desenvolvidas as seguintes funcionalidades: cadastro de usuários, cadastro de aplicações, questionário de satisfação e relatório de avaliação.

Existem dois tipos de usuários nesta aplicação: usuário administrador, responsável pelo cadastro de usuários e de aplicações, bem como geração de relatório de avaliação; usuário avaliador, cuja função é responder o checklist disponibilizado na aplicação. Este checklist é composto por vinte e cinco questões acerca de aspectos, princípios e diretrizes de usabilidade de interfaces. Para cada um dos questionamentos, existem duas opções de respostas (sim ou não).

O presente estudo de caso foi conduzido a partir de dois objetivos principais: (i) analisar a aplicação do Framework UEF-WEB para verificar a usabilidade das interfaces construídas sob o ponto de vista dos usuários; (ii) analisar a adoção do Framework UEF-WEB para observar, sob a perspectiva da equipe de desenvolvimento, a percepção de utilidade e facilidade de uso deste framework.

Para atingir o primeiro objetivo, foi aplicado um questionário de satisfação a vinte usuários da aplicação AvWeb. Este questionário só foi disponibilizado após a realização de tarefas e interação dos usuários com a aplicação. Neste questionário, os usuários foram convidados a avaliar o nível de usabilidade percebido em relação à aplicação AvWeb através dos critérios de eficácia, eficiência e satisfação discutidos na 
norma ISO 9241-210 (ISO, 2010). Para cada um desses critérios de usabilidade, os usuários atribuíram um dos seguintes níveis de avaliação: alto, médio, baixo ou muito baixo. Os resultados obtidos neste questionário são apresentados na Figura 1.

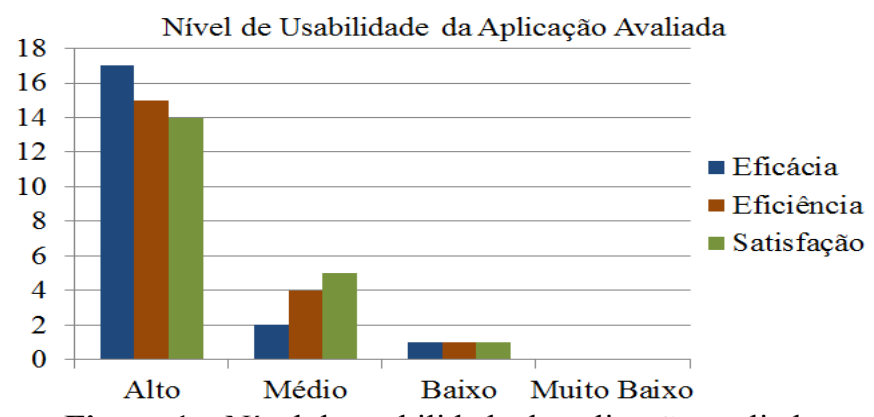

Figura 1 - Nível de usabilidade da aplicação avaliada.

Com base nas informações da Figura 1, foi possível observar que os usuários avaliaram positivamente a qualidade de uso da aplicação AvWeb, tendo em vista que dezessete, quinze e quatorze dos vinte usuários atribuíram o nível de avaliação "alto" para os critérios de usabilidade eficácia, eficiência e satisfação, respectivamente. Apenas um usuário atribuiu o nível de avaliação "baixo" para cada um dos critérios. Nenhum dos usuários, entretanto, concedeu o nível de avaliação "muito baixo" para os critérios apresentados.

No que diz respeito ao segundo objetivo, foi aplicado um questionário de satisfação aos cinco membros da equipe de desenvolvimento. Este questionário, composto por três questões, foi respondido por todos os membros após a construção da aplicação AvWeb. Para as duas primeiras questões, foi empregada uma escala Likert de quatro níveis e sem um valor neutro intermediário, pois este valor, em algumas ocasiões, não contribui para determinar o posicionamento (positivo ou negativo) do participante sobre os questionamentos apresentados.

A primeira questão diz respeito à percepção de utilidade e a segunda questão se refere à facilidade de uso do framework na visão dos integrantes da equipe de desenvolvimento. $\mathrm{O}$ terceiro questionamento, por outro lado, procurou observar a disposição em utilizar o framework proposto em novos projetos de interfaces de aplicações $W e b$. Os resultados deste questionário são apresentados na Figura 2.

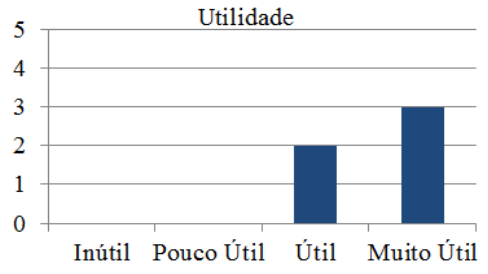

(a)

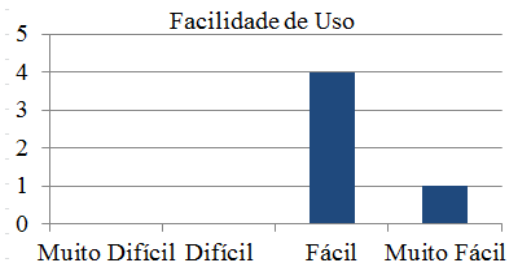

(b)

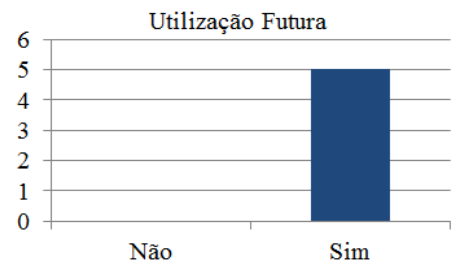

(c)

Figura 2 - Percepção de utilidade, facilidade de uso e interesse no Framework UEF-WEB.

Tendo por base a Figura 2, foi possível constatar que os membros da equipe de desenvolvimento responderam positivamente aos questionamentos relativos à utilidade e facilidade de uso do Framework UEF-WEB. Toda a equipe de desenvolvimento não somente reconheceu a importância deste framework para o processo de construção de interfaces com maior qualidade de uso, como também relatou facilidade ao utilizá-lo. Por fim, todos os membros da equipe manifestaram interesse em empregar o framework proposto em novos projetos de aplicações $W e b$. Na próxima seção, serão apresentadas as considerações finais deste artigo. 


\section{Considerações Finais}

Como se observou, a demanda por aplicações $W e b$ cresceu rapidamente para atender às necessidades das pessoas e organizações. Essa expansão vem sendo percebida não somente em relação à quantidade, mas, também, na diversidade e nível de complexidade das aplicações. A crescente importância dessas aplicações para a sociedade tem requisitado a utilização de boas práticas no processo de desenvolvimento de softwares, no intuito destes serem entregues dentro do prazo e orçamento definidos, com elevado grau de qualidade e facilidade de manutenção. À luz do discutido, o atendimento aos requisitos de usabilidade e de acessibilidade constitui um desafio a mais e crítico para as organizações, pois agregam valor a serviços e produtos cada vez mais utilizados na contemporaneidade como o e-learning e e-commerce. Assim, se torna imprescindível desenvolver aplicações satisfatórias às necessidades dos usuários e em conformidade a estes requisitos.

Diante disso, este artigo propôs o Framework UEF-WEB como instrumento de suporte à usabilidade para auxiliar as organizações a adotar, de forma sistemática e facilitada, recursos de usabilidade no processo de planejamento, desenvolvimento e avaliação de interfaces de aplicações $W e b$. Este framework foi construído a partir de pesquisa bibliográfica e análise crítica de contribuições relacionadas à Engenharia de Usabilidade identificadas na literatura.

Os resultados do estudo de caso realizado nesta pesquisa forneceram indícios positivos da viabilidade de utilização deste framework como instrumento de apoio à usabilidade no desenvolvimento de aplicações $W e b$ ergonômicas, de modo a incentivar a sua adoção em outros projetos de interfaces. Como possibilidade de trabalhos futuros, tem-se a construção da ferramenta "UEF-WEB Tool" para suportar as fases, atividades, recursos e artefatos estabelecidos no Framework UEF-WEB.

\section{Referências}

AHMAD, W. F. W; SULAIMAN, S; JOHARI, F. S. Usability Management System (USEMATE): A Web-Based Automated System for Managing Usability Testing Systematically. In: International Conference on User Science Engineering (i-USEr), 2010.

BANGOR, A; KORTUM, P; MILLER, J. Determining what individual SUS score mean: adding an adjective rating scale. In: Journal of Usability Studies, v. 4, issue 3, pp. 114-123, 2009.

BROOKE, J. SUS - A quick and dirty usability scale. 1986. Disponível em: $<\mathrm{http}$ ://www.usabilitynet.org/tools/r_questionnaire.htm>. Acesso em: 25 ago. 2014.

CONTE, T. U. Técnica de inspeção de usabilidade baseada em perspectivas de projeto Web. Rio de Janeiro: UFRJ/COPPE. Tese de Doutorado. Universidade Federal do Rio de Janeiro, 2009.

CYBIS, Walter; BETIOL, Adriana Holtz; FAUST, Richard. Ergonomia e usabilidade: conhecimentos, métodos e aplicações. 2. ed. São Paulo: Novatec, 2010.

FERNANDES, P; CONTE, T; BONIFÁCIO, B. WE-QT: A Web Inspection Technique to Support Novice Inspectors. In: Brazilian Symposium on Software Engineering, 2012.

FERRE, Xavier; BEVAN, Nigel. Usability Planner: A Tool to Support the Process of Selecting Usability Methods. In: INTERACT 2011, Part IV, LNCS 6949, pp. 652-655. Springer-Verlag, 2011. 
GALITZ, W. O. (2003). The Essential Guide to User Interface Design: An Introduction to GUI Design Principles and Techniques. 2 ed. John Wiley \& Sons: New York, 2003.

GIMENES, I. M. S; HUZITA, E. H. M. Desenvolvimento Baseado em Componentes. Rio de Janeiro: Ciência Moderna, 2005.

HART, D; PORTWOOD, D. M. Usability Testing of Web Sites Designed for Communities of Practice: Tests of the IEEE Professional Communication Society (PCS) Web Site Combining Specialized Heuristic Evaluation and Task-Based User Testing. In: IEEE International Professional Communication Conference (IPCC), 2009.

HITZ, M; LEITNER, G; MELCHER, R. Usability of Web Applications. In: Kappel, G; Proll, B; Reich, S; Retschitzegger, W. (Eds.). Web Engineering: The Discipline of systematic Development of Web Applications, Chapter 11, John Wiley \& Sons, 2006.

INSFRAN, E; FERNANDEZ, A. A Systematic Review of Usability Evaluation in Web Development. In: Proceedings of Second International Workshop on Web Usability and Accessibility (IWWUA 2008), v. LNCS 5176 - Advances in Web Information Systems Engineering - WISE 2008 Workshops, pp. 81-91, New Zealand, 2008.

International Standards Organization (ISO). ISO 9241-210. Human-centred design for interactive systems. Geneva: International Standards Organization, 2010.

MATERA, M; RIZZO, F; CARUGHI, G. Web Usability: Principles and Evaluation Methods. In: Mendes, E; Mosley, N. (Eds.). Web Engineering, Chapter 5, New York, Spring Verlag, 2006.

NIELSEN, J; LORANGER, H. Usabilidade na Web. 2. ed. Rio de Janeiro: Elsevier, 2007.

NIELSEN, J. Heuristic evaluation. In: NIELSEN, J; MACK, R (Eds.). Usability inspection methods, Heuristic Evaluation. New York: John Wiley \& Sons, 1994.

PRESSMAN, Roger. S. Engenharia de Software. Tradução de Ariovaldo Griesi e Mario Moro Fecchio. 7. ed. Porto Alegre: AMGH, 2011.

PRESSMAN, Roger S; LOWE, David. Engenharia Web. Tradução de Daniel Vieira. Rio de Janeiro: LTC, 2009.

ROCHA, H. V; BARANAUSKAS, M. C. C. Design e Avaliação de Interfaces Humano-Computador. Campinas: NIED/UNICAMP, 2003.

SCHNEIDER, H. N. Interface de Software Educacional: a questão da usabilidade. In: CRUZ, M. H. S. Pluralidade dos saberes e territórios de pesquisa em educação sob múltiplos olhares dos sujeitos investigadores. Aracaju: Editora UFS, 2008, p. 199231.

SKOV, M; STAGE, J. Supporting problem identification in usability evaluations. In: Proceedings of the 17th Australia conference on Computer Human Interaction: Citizens Online: Considerations for Today and the Future, v. 122, pp. 1-9, Austrália, 2005.

SOMMERVILLE, Ian. Engenharia de software. Tradução de Selma Shin Shimizu Melnikoff, Reginaldo Arakaki e Edílson de Andrade Barbosa. 8. ed. São Paulo: Pearson, 2007.

TOMHAVE, B. L. Alphabet Soup: making sense of models, frameworks and methodologies. 2005. Disponível em: $<$ http://www.secureconsulting.net/Papers/Alphabet_Soup.pdf $>$. Acesso em: 05 jan. 2015. 